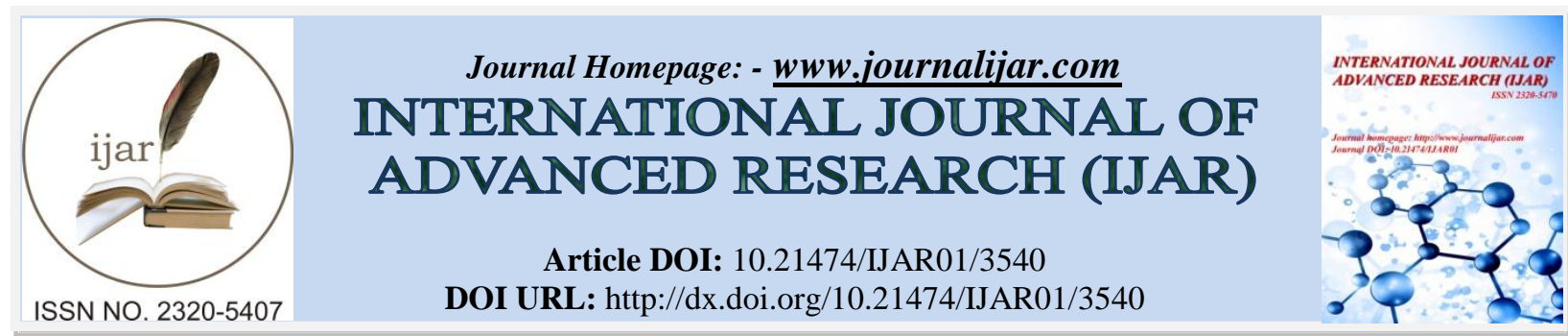

RESEARCH ARTICLE

\title{
LES STRUCTURES DE GESTION DES DESTINATIONS TOURISTIQUES ET LEURS FONCTIONS FACE AUX DEFIS DE LA COMPETITIVITE : UNE SYNTHESE DE LA LITTERATURE.
}

Achaba Allal et Bendou Abdelaziz.

Enseignants Chercheurs à l'Ecole Nationale de Commerce et de Gestion Agadir, Université Ibn Zohr (Maroc).

\section{Manuscript Info}

Manuscript History

Received: 14 January 2017

Final Accepted: 16 February 2017

Published: March 2017

Key words:-

Compétitivité, destination touristique, compétitivité des destinations

touristiques, structure et fonctions de gestion, organe de gestion des

destinations, management de destination.

\section{Abstract}

Il n'est plus d'intérêt de rappeler l'importance du tourisme pour le développement socioéconomique des pays et des territoires. Le secteur devenant primordial dans les stratégies de développement de nombreux pays va attirer depuis près de trois décennies l'intérêt des chercheurs de tous les horizons qui vont chercher à le comprendre, l'analyser et identifier les facteurs clés de son développement. De même, le fort taux de croissance du secteur, sa forte résilience et résistance aux crises ainsi que les perspectives très encourageantes de son développement futur vont enflammer la course à la compétitivité des destinations touristiques, devenues les unités de base et centrales dans cette quête de compétitivité vont être au centre des politiques et des stratégies de développement touristiques, mais aussi, faire foisonner la recherche scientifique sur les conditions, les formes et les dimensions de cette compétitivité et sur les défis qu'elle impose et les formes, rôles et fonctions des structures de management de ces destinations pour y répondre efficacement et assoir la compétitivité des destinations. Malgré, le caractère très récent de la recherche managériale sur ces différentes questions un nombre assez important de recherches ont été produites et un corpus théorique est entrain de se constituer et de s'établir.

Cet article, de nature exploratoire, se propose d'étudier, à l'aune de la littérature, le rôle et les fonctions des structures de gestion des destinations touristiques dans le cadre des nombreux défis imposés par la recherche de la compétitivité dans un marché touristique mondial évolutif, mutants et hautement concurrentiel. Plus particulièrement et à travers une synthèse de la littérature existante tant sur la compétitivité et ses implications pour les destinations touristique que sur les théories et modèles de management des destinations touristiques, nous cherchons à faire un état de la littérature sur ces questions et à relever le degré d'intégration des défis posés par la compétitivité dans les rôles et fonctions des structures de gestion des destinations touristiques.

Copy Right, IJAR, 2017,. All rights reserved.
Corresponding Author:- Achaba Allal Et Bendou Abdelaziz. Address:- Enseignants Chercheurs à l'Ecole Nationale de Commerce et de Gestion Agadir, Université Ibn Zohr (Maroc). 


\section{Introduction:-}

Depuis que le tourisme est reconnu comme secteur économique important, la question de la compétitivité des destinations touristiques est devenue à l'ordre du jour et intéresse aussi bien les gouvernements, les chercheurs que les organismes internationaux. En effet, le secteur touristique de part sa croissance continue et ses apports aux équilibres socioéconomiques de nombreux pays tant développés, émergents qu'en voie de développement est devenu un objet majeur au centre des politiques de développement prônées par les gouvernements et les organismes internationaux qu'un objet central dans les axes de recherche de plusieurs chercheurs à travers le monde. La nature mondialisée et hautement concurrentielle du marché touristique international fait que le la réussite dépend largement de la position de ce secteur dans le marché international du tourisme (Gomezelj et Mihalic, 2008). De même, au centre du secteur, les destinations se pointent comme les unités centrales et importantes de l'analyse et de la mise en œuvre des politiques et stratégie touristiques. Et le succès d'une destination dans le tourisme international dépend en grande partie de sa compétitivité (Enright et Newton, 2004). Ainsi, La compétitivité des destinations devient un objet d'études central tant des chercheurs en tourisme que des organismes internationaux intéressés par le secteur qui s'intéressent tous à comprendre les dimensions et les enjeux de cette compétitivité et à étudier les aptitudes des entreprises, des territoires et des pays à soutenir la concurrence sur un marché mondial de plus en plus évolutif, saturé et intégré et à discuter plus particulièrement des fonctions et taches fondamentales que doivent remplir les structures de management des destinations pour maintenir et améliorer leur compétitivité (Gomezelj et Mihalic, 2008).

Si la question des effets des structures organisationnelles pour la performance et le succès des organisations est un sujet largement discuté et débattu dans les sciences du management, la pertinence des structures organisationnelles pour la compétitivité des destinations touristiques est un sujet récent et qui depuis son initiation par Ritchie et Crouch en 1993 est devenu au centre de l'intérêt de la recherche scientifique sur le tourisme. Cette position centrale prise par cette thématique émane de la congruence de plus en plus constatée de la perception des destinations, par les chercheurs et les gouvernements, comme les unités compétitives du tourisme sur les quelles doivent ce centrer les questions de recherche et d'analyse et de mise en œuvre des actions de promotion et de développement visant l'amélioration de la compétitivité. Poser la question des effets des structures organisationnelles du management des destinations touristiques sur la compétitivité des destinations touristiques se heurte à plusieurs difficultés liées en premier lieu à la nature complexe et polysémique des concepts de compétitivité et de destination touristique et en second lieu à la problématique même de ces structures de gestion et de leurs fonctions.

Dans cet article de nature exploratoire nous chercherons à mettre en lumière, sur la base d'une étude documentaire relative aux différentes avancées théoriques sur la question, les fonctions, taches et rôles que doivent assurer les structures de gestion des destinations pour faire face aux défis imposés par la compétitivité dans le secteur touristique. Cette quête nous mènera tout d'abord à s'interroger sur la question de la compétitivité des destinations touristiques et relever les principaux défis qu'elle impose aux organes de gestion des destinations touristique pour ensuite s'interroger sur ces différentes structures et leurs fonctions et enfin dégager les conclusions et les perspectives.

\section{Sur les Destinations Touristiques et leur Competitivite:-}

Dans cette section nous tenterons de mettre en relief tous les aspects de la littérature relatifs aux concepts clés de notre recherche à savoir notre champ d'investigation - les destinations touristiques, le concept de compétitivité et celui de compétitivité des destinations touristique et les théories et modèles qui ont tenté de l'expliquer pour ensuite essayer identifier les principaux défis que cette compétitivité impose au management des destinations.

\section{La destination touristique, un concept complexe et insuffisamment definie:-}

Le succès du tourisme est étroitement associé au mot destination (Kadri et al., 2011). Toutefois ce concept de destination reste difficile à définir (Haywood, 1986). En effet, il existe une multitude de définition qui ont été données à la destination touristique. La littérature d'origine institutionnelle la définit généralement en référence à un espace géographique relativement cohérent, doté d'une certaine autonomie décisionnelle et présentant des caractéristiques attractives pour les visiteurs extérieurs auxquels des produits adaptés sont proposés (Commission européenne, 2000). L’Organisation Mondiale du Tourisme (OMT) la définit comme «tout territoire touristique accessible, incluant des infrastructures d'hébergement, de transport, ainsi que des activités, animations et services, constitue une destination. Cette destination, qui peut être définie par des limites physiques, thématiques ou administratives, adopte un ensemble d'images et de qualités distinctives qui lui donnent une identité.». Si l'on se réfère à présent à la Resource-based view (RBV), une destination peut être définie comme un ensemble de 
ressources productives et humaines (Penrose, 1959) dont le stock disponible à un instant $t$ résulte d'une économie d'accumulation des ressources (Cool, 2000) réalisée au fil des siècles par les générations successives (Dierickx et Cool, 1989). De même de nombreux chercheurs émanant de plusieurs disciplines ont tenté de cerner ce concept en lui apportant des définitions. Ainsi, GEE et al (1989) considèrent que les destinations touristiques représentent des domaines spécifiques que les voyageurs choisissent de visiter et où ils passent une période de temps significative. Mathieson et Wall's (1992) avancent qu'une destination touristique correspond à un endroit qui présente des caractéristiques qui sont connues par un nombre suffisant de visiteurs potentiels et qu'ils considèrent comme une identité qui attire les voyageurs quelques soient les attractions d'autres endroits. Pour Jenkins et Tosun (1996) les destinations touristiques sont des domaines ayant des caractéristiques naturelles ou créées par l'homme et qui attirent les visiteurs locaux et étrangers. De leur coté, Morant et al (1996) conçoivent la destination touristique comme une combinaison d'éléments interdépendants (ressources, infrastructure, transports, ...) qui produisent de la satisfaction des touristes. Kim (1998), lui, présente la destination touristique comme un produit exceptionnellement complexe de l'industrie du tourisme qui comprend, entre autres, le climat, l'infrastructure et de la superstructure (services et attributs naturels et culturels). D'autres auteurs adoptant des points de vue très étroits considèrent la destination comme une localité (Scaramuzzi, 1993), une station touristique, une zone touristique ou une région (Sancho, 1998).

De toutes ces définitions, il ressort qu'il ya un certain nombre de points communs partagés par les auteurs notamment la référence à l'aspect géographique (endroit, lieu, domaine, territoire, zone, région, localité), à l'aspect du fonctionnement en terme économique (marché, produits, services), l'aspect ressources et disponibilités (climat, infrastructures, ressources naturelles et culturelles,...) et à l'aspect psychologique (motivation du touriste et visiteurs).

Toutefois, malgré cette multitude de définition nous pouvons avancer que le concept de destination, au cœur de la compréhension du phénomène qu'est le tourisme (Botti et Séraphin, 2011), possède également un caractère complexe et souffre d'une insuffisance définitionnelle et de théorisation (Boualem et al, 2011) et ne fait pas l'objet d'un consensus commun, unanime et complet de tous les chercheurs. Il est ainsi, claire que le concept de destination pris dans sa totalité et sa complexité pose un problème de définition et d'approche. Une lecture de la littérature nous informe que le concept de destination est devenu en quelque sorte un mot-valise mythique (Hazebroucq, 2009 : 8) dont la signification est fortement corrélée à des considérations contextuelles (Kadri et al., 2011). Et cela est dû, selon les chercheurs, au fait de la domination scientifique de deux principales disciplines qui sont la géographie et le marketing qui ont été les premières à s'intéresser au concept de destination et qui l'on adapté à leur objet et imposer leur façon de l'aborder. La géographie, ayant été la première à s'accaparer le concept et l'étudier, a mis toujours en avant la dimension spatiale. Ainsi, les définitions telles que celles de l'Équipe MIT (2005, p. 11) et de Hayllar et al. (2008), montrent clairement le poids de la variable spatiale dans l'interprétation de ce concept (kadri et al, 2011). Pour l'Equipe MIT, «La destination est un lieu d'interactions territoriales qui relient ensemble les données socioculturelles, naturelles, patrimoniales et techniques» et pour Hayllar et al., « l'étude des territoires touristiques concerne d'abord l'étude des espaces.». Ainsi, nous pouvons relever que le concept de destination tel que abordé par cette discipline est resté, compte tenu de fait que les pratiques touristiques supposent mobilité et déplacement, attaché à une dimension spatiale malgré la nature de totalité et de complexité du phénomène touristique (Violier, 2009). Cet auteur, revient ensuite pour critiquer le réductionnisme de cette approche qu'il juge positiviste dans le sens où elle ne définie le lieu touristique que par la présence d'objet naturels ou culturels; alors que pour lui, la présence d'objets ne suffit pas à assurer la présence des touristes. Selon cet auteur « la destination serait ce que les touristes perçoivent comme l'espace où ils mettront en œuvre des pratiques qui définissent alors le territoire touristique » (Violier, 2009). C'est dans le même sens que Caccomo (2006, p.136) avance que «c'est la fréquentation qui confère sa valeur à la destination» ou Ceriani et al. (2008) qui considèrent qu'un «lieu est touristique par les touristes qui le pratiquent et que, dialogiquement, il correspond plus ou moins au projet formé par les touristes». C'est une fois que la discipline du marketing va s'intéresser au concept de destination qu'un élargissement de sa conception va se constater. Sans occulter la dimension spatiale cette discipline va approcher la destination touristique comme un produit auquel peuvent s'appliquer les stratégies et les techniques de marketing applicables aux produits industriels. C'est déjà un géographe en la personne de Richard Butler qui a été le premier a présenter en 1980 un modèle, inspiré de la théorie du cycle de vie du produit, mettant en perspective la naissance et le déclin de la destination en fonction de l'évolution du nombre des visiteurs dans le temps. D'autres auteurs vont suivre cette nouvelle voie ouverte par Buttler et s'intéresser à la destination comme produit, notamment Tocquer et Zins (1999) qui définissent la destination touristique comme un type de produits et services touristiques issus de nombreux opérateurs privés et publics et Davidson et Maitland (2002) qui considèrent la destination comme un lieu de production de produits touristiques concentrant l'attention et les motivations des visiteurs. D'autres auteurs 
comme Kye-Sung (1990), Etchner et Ritchie (1991), Stabler (1988), Telisman-Kosuta (1989) et Gallarza et al. (2002), quand à eux vont aborder la définition de la destination à partir de l'image que cette dernière renvoie à ses visiteurs. Dans cette perspective, la destination peut être un site, un village, une ville, une région, un pays ou une zone et la territorialité de la destination dépendra à la fois de l'effort de communication entrepris par cette dernière en sus des expériences vécues. De ce point de vue, le concept acquiert une dimension vitale dans la construction identitaire du territoire, dans la mesure où «la destination devient un lieu qui prend la dimension de destination», la marque de ce lieu (Gibson et Tiard, 1999, p. 79).

Comprendre le concept de destination touristique implique donc de faire la synthèse entre une approche géographique (la destination est avant tout un lieu qu'un ou plusieurs touristes ont décidé de visiter) et une approche marketing pour laquelle la destination est un territoire commercialisé. Dans cette perspective, la destination est appréhendée comme un territoire qui peut-être en lui-même un produit touristique, c'est-à-dire un ensemble de services supports (hôtellerie, restauration, transport...) qui gravitent autour d'éléments attractifs et qui, dans son ensemble, peut répondre aux besoins d'un segment de touristes (Botti et al., 2008).

Les travaux modernes issus des disciplines du management et de la gouvernance quant à eux ont élargi le champ de conceptualisation de la destination pour la rehausser au stade de système (Beritelli et al, 2007, Ruhanen et al, 2010, Laws et al. 2011, Zhang et Zhu, 2014). A ce titre Hazbroucq (2007) considère la destination comme «un « macroproduit» touristique qui repose sur un système complexe composé d'acteurs hétérogènes et disséminés sur le territoire» (Hazbroucq, 2007, p.121). Scott et al., (2008) avancent, de leur coté, qu'une destination peut être considérée comme «un groupe d'acteurs inter-reliés intégrés dans un réseau social. Ce groupe d'acteurs, malgré leur indépendance sont interdépendants dans leur recherche de la réponse aux besoins des touristes et visiteurs et participent tous à la fourniture de l'expérience clients. L'interaction de ces acteurs est complexe, dynamique et soumise à des chocs externes».

\section{La compétitivité, un concept polysémique:-}

Comme l'affirment Tixier et Mathe (1996), le souci de compétitivité occupe une position centrale parmi les éléments fondateurs des orientations politiques de l'entreprise. Pour cette raison, toute entreprise dont les activités veulent s'inscrire dans la durée doit intégrer cette notion dans son vécu quotidien. Le concept de compétitivité est un concept central dans la recherche économique et managériale. Issu en premier lieu du monde de l'entreprise où il s'appliquait à un processus industriel, à un produit ou à une firme et où il désignait la capacité de l'entreprise à réaliser des gains de parts de marché par rapport à ses concurrents ou a sa "capacité à réaliser des performances supérieures à la moyenne » (Marniesse et Filipiak, 2003) et comme « résultant de la capacité de l'entreprise à maintenir ses performances sur le long terme » (Muchielli, 2002). La compétitivité de l'entreprise est ainsi fonction de ses performances commerciales et se définit par rapport à sa position sur les marchés vis-à-vis de ses principaux concurrents. La notion de compétitivité fait allusion directe à celle de concurrence. Ainsi, être compétitif, c'est «être apte à affronter dans des conditions favorables la concurrence qui s'exerce dans un domaine de la vie économique et sociale».

Sur le plan des corpus théoriques managériaux ayant admis l'importance de la compétitivité, notre examen de la littérature nous montre que les principales approches se sont plus focalisées sur l'identification et l'analyse des sources et des facteurs de la compétitivité des entreprises. Ces corpus peuvent être distingués en deux principales approches : l'approche unidimensionnelle et l'approche pluridimensionnelle. La première approche est représentée par les travaux du célèbre bureau de consulting le Boston Consulting Group (B.C.G.) dont les adeptes voyaient en les coûts la principale source de compétitivité. En effet, pour le BCG, «dans un milieu concurrentiel, l'entreprise compétitive est celle qui a les coûts les plus bas, c'est à dire celle qui a su parvenir à l'utilisation la plus efficace des facteurs pour des coûts de facteurs équivalents et ce par le biais de l'expérience » (BCG, 1988). On peut déduire de cela que pour ce courant la compétitivité d'une entreprise se traduit exclusivement par la compétitivité de ses produits. Cette approche a été vivement critiquée par les chercheurs qui la considéraient d'une part réductrice pour avoir ramené la compétitivité à la seule dimension coût alors que d'autres dimensions telles que la qualité des produits, l'image du marque, les facilités de paiement sont à envisager dans une lutte concurrentielle et d'autre part sa non pertinence en matière d'analyse des coûts qu'elle aborde de manière séquentielle sans tenir compte des liaisons entre les activités et leur impacts sur ceux-ci. Ainsi, à la dimension coûts il est judicieux de tenir compte d'autres dimensions hors coût pour représenter la compétitivité. De l'autre coté, la lecture de la littérature concernant l'approche pluridimensionnelle nous renseigne que les tenants de cette approche ont développé plusieurs schémas d'analyse des sources, des facteurs et des déterminants de la compétitivité. En premier lieu, M. Porter 
(M.E. PORTER 1985) dans son modèle de la chaîne de valeur a mis l'accent sur l'importance des liaisons entre les différentes activités comme sources de l'avantage concurrentiel de l'entreprise. De leur coté les adeptes de l'approche basée sur les ressources (RVC), (Wernerfelt, 1984 ; Rumelt, 1984 et Barney,1986 et 1991), considèrent que l'avantage concurrentiel est à rechercher en interne. Ainsi, en concevant l'entreprise comme un portefeuille de ressources et non comme un portefeuille de Produits/Marché les tenants de l'approche ressources et compétences avancent que ce ne sont plus les besoins des clients qui déterminent la stratégie, mais se sont les ressources et les compétences que l'entreprise possède et l'acquisition de l'avantage compétitif provient de la combinaison des ressources au sein de l'entreprise et leur traduction en termes de stratégie à mettre en œuvre sur les marchés des biens. Cette approche considère que les entreprises sont capables d'accumuler des ressources et des compétences qui se transforment en avantage sur les concurrents si elles sont rares, créatrices de valeur, non substituables et difficiles à imiter (Barney, 1991; Dierickx et Cool, 1989). Ces ressources désignent généralement des actifs possédés de façon permanente ou contrôlés par l'entreprise en vue de concevoir et de mettre en œuvre sa stratégie. Barney (1991) les distingue en trois principales catégories: le capital physique, le capital organisationnel et le capital humain. Plus tard, trois autres catégories se sont venues s'ajouter en l'occurrence les ressources financières, les ressources technologiques (savoir-faire, brevets...) et les ressources réputationnelles (marques, notoriété...). Les compétences désignent de ce fait, la capacité organisationnelle de l'entreprise à pouvoir déployer ces ressources ou actifs sous forme de combinaisons efficaces et efficientes pour atteindre son objectif.

Certaines autres approches se sont attaquées à la question à travers un autre angle de vision notamment en mettant l'accent plus sur le processus causal de création de l'avantage compétitif plutôt que sur les ressources, les compétences ou les activités. A ce titre Lawrence et Lorsh (1986) estiment que la compétitivité d'une entreprise est principalement associée au degré de flexibilité de sa structure et sa capacité d'adaptation aux exigences de son environnement. Pour ces auteurs, rester compétitif exige une grande capacité d'adaptation aux changements de l'environnement qui se manifeste à travers d'une part l'adaptation de la structure organisationnelle, l'adaptation des compétences des dirigeants et l'insertion compétitive de l'entreprise dans son environnement. De leur coté, Hamel et Prahalad (1994), stipulent, contrairement aux avancées de porter qu'ils ont jugé trop défensives et à usage exclusif des entreprises dominantes et aux travaux de l'approche ressources qu'ils ont jugé trop centrés sur l'interne négligeant les mutations de l'environnement et des bases de la concurrence mais aussi de l'approche basée sur l'adaptation prônée par Lawrence et Lorch, que la compétitivité suppose une vision volontariste et offensive de la stratégie d'entreprise. Ce qu'ils qualifient d'intention stratégique (Hamel et Prahalad, 1994). En effet, selon ces auteurs, pour réussir et détenir un avantage compétitif, une entreprise ne doit pas s'adapter à son environnement mais le changer, le transformer à son profit. L'art du stratège est de modifier les règles du jeu concurrentiel et non de s'y conformer et le changement n'est plus une contrainte mais un objectif. Pour ces deux auteurs la stratégie ne ce conçoit pas sans intentionnalité et l'entreprise doit avoir une ambition permanente de compétitivité partagée par tous ses membres, non directement reliée aux ressources actuelles détenues et permettant d'avoir un point de repère face à un environnement turbulent tout en créant une tension créatrice de valeur et de changement basée sur une vison claire des dirigeants permettant d'identifier les ressources et compétences manquantes afin d'atteindre le développement future souhaité.

Cette notion de compétitivité après avoir conquit le monde de l'entreprise va voir s'élargir son horizon dans les années 1980 pour s'appliquer à d'autres entité et dimensions, notamment le secteur, la branche, le territoire, la région et la nation. En effet, s'il est possible de s'interroger sur la compétitivité d'une entreprise, il est aussi possible de s'interroger sur celle d'une branche industrielle, d'un territoire ou encore de l'économie d'une nation, etc. Ainsi, de plus en plus va émerger une approche que l'on peu qualifier de macroéconomique de la compétitivité qui s'intéresse aux territoires et à la nation tout entière. Et de nombreuses définitions vont être données par les chercheurs à cette compétitivité. Ainsi, Debonneuil et Fontagne, 2003, p. 8) définissent la compétitivité d'une nation ou d'un territoire comme la "Capacité à améliorer durablement le niveau de vie de ses habitants (...) et à leur procurer un haut niveau d'emploi et de cohésion sociale». Dans la même veine D'Andrea Tyson la conçoit comme l' "Aptitude à produire des biens et des services qui satisfont au test de la concurrence sur les marchés internationaux et à augmenter de façon durable le niveau de vie de la population ». de sa part l'OCDE présente la compétitivité comme «la latitude dont dispose un pays évoluant dans des conditions de marché libre et équitable pour produire des biens et services qui satisfont aux normes internationales du marché tout en maintenant et en augmentant simultanément les revenus réels de ses habitants dans le long terme ».

Cette transposition du terme «compétitivité» pour qualifier des entités géographiques a été très critiquée par plusieurs auteurs, en particulier par le prix Nobel Paul Krugman et M.E. Porter. Pour Krugman, «parler de la 
compétitivité des pays n'aurait pas de sens » et « la plupart de ceux qui utilisent le terme de compétitivité ne s'y sont même pas arrêtés pour réfléchir à la chose »(Krugman, 2001, p. 20). De son coté, Porter estime que «chercher à expliquer la 'compétitivité' au niveau national est incorrecte. Ce qu'on doit comprendre, ce sont les déterminants de la productivité et le taux de croissance de la productivité. Pour trouver des solutions, on devrait focaliser, non pas sur l'ensemble de l'économie, mais plutôt sur des industries spécifiques ou encore des segments d'industrie" (Porter, 1990). Et il ajoute des années plus tard qu' «il n'y a même pas de définition admise du terme compétitivité appliquée à un pays. La notion de compétitivité est claire quand elle s'applique à une entreprise pas quand elle s'applique à un pays » (Porter, 1999, p.167). La majorité des auteurs ayant critiqué cette transition remettent en cause principalement l'incompatibilité des définitions de la compétitivité des territoires et des nations, elles mêmes porteuses de contradictions, avec les types de compétitivité de l'entreprise mais surtout les difficultés de l'utilisation d'outils conceptuelles identiques et les difficultés méthodologique d'établir des critères identiques pour mesure (Delaplace, 2011). Malgré tout cela, Aiginger, (2006) estime que même en présence de certaines controverses sur le concept, les travaux théoriques et les débats qui entourent la compétitivité des nations ont permis de faire émerger, une définition consensuelle

Somme toute, notre examen de la littérature traitant du concept de compétitivité nous révèle la nature polysémique, ambigüe, complexe et multidimensionnelle du concept et l'absence d'une définition unique et unanime du terme. Marniesse et Filipiak (2003) considèrent que «la compétitivité est une notion encore mal cernée». Porter (1990) stipule que «son ambiguïté découle de l'existence d'une variété de définitions qui rend difficile une définition complète et indiscutable de la compétitivité ». Quand à Scott et Lodge (1985), ils expliquent cette complexité par la nature multidimensionnelle et relative du concept même de compétitivité. Par ailleurs, il ressort aussi que le concept est relatif et dynamique. Relatif du fait qu'il change avec le niveau de l'analyse menée (nation, secteur, entreprise), le bien analysé (bien homogène ou différencié), et enfin l'objectif de l'analyse et dynamique dans le sens où la compétitivité n'est pas un concept statique et est étroitement lié aux conditions économiques du pays ainsi que les conditions du marché international.

\section{La compétitivite des destinations touristiques : entre recherche académique et modèles opérationnels:-}

Malgré que le tourisme soit devenu un secteur économique très important dans la compétitivité des pays et des territoires, il est paradoxal de relever qu'il est resté un des parents pauvre de la recherche scientifique et un domaine d'étude récent. Certes, les difficultés à l'appréhender compte tenu de l'absence d'une définition unanime et universelle, les mythes et les préjugés qui lui sont associés et les difficultés de mesure de sa productivité ont fait que les économistes ont longtemps tardé à s'y intéresser et à lui accorder la place qu'il mérite comme secteur économique à part entière et comme sujet de recherche. C'est ainsi, que c'est à partir des années 1970 que les économistes et après eux les géographes ont commencé à l'étudier après que les services et plus particulièrement le tourisme sont devenus très important dans les PIB des pays développés. Les sciences de gestion vont emprunter la voie à ces disciplines mais plus tardivement vers la fin des années 1980. Reconnu comme multiforme et multisectoriel est donc multi-produit et multi-métier (Botti et Peypoch), le tourisme nécessite pour sa compréhension et son développement de faire appel à plusieurs facettes du management (stratégie, politique, marketing, gestion des ressources humaines, gestion financière,...). Cet engouement vers le secteur va rapidement fait naitre un foisonnement de travaux de recherche d'abord en Amérique du Nord (Bornhorts, Brent Richie, et Sheehan, 2010; Minguzzi, 2006) puis en Europe (Escadafal (1997), Dewailly (2006), Lozato Giotart et Balfet (2004) et Maunier (2007)). Rapidement l'intérêt pour la gestion et la compétitivité des destinations touristiques va passer au premier plan de l'intérêt des chercheurs après que celles-ci vont être reconnues d'une part, comme les unités de bases sur lesquelles repose la compétitivité du tourisme en général et des territoires en particuliers et d'autre part, de la prise de conscience que la concurrence internationale dans le secteur se fait de plus en plus entre destinations et non entre entreprises. En effet, Dwyer et al. (2000) nous enseignent qu'il est très utile pour l'industrie et le gouvernement de comprendre quels sont les points forts et les points faibles d'un pays par rapport à ses concurrents en matière de tourisme et de savoir comment et pourquoi la compétitivité d'une destination change. Teece et al. (2008), pour leur part, soulignent que la nature dynamique de la concurrence en matière de tourisme exige des destinations de combiner et gérer leurs ressources touristiques de manière à développer un avantage concurrentiel. Par conséquent, les destinations doivent faire face au défi de gérer et organiser leurs ressources rares de manière efficiente afin de fournir une expérience de vacances supérieure à d'autres expériences dans d'autres destinations (Cracolici et Nijkamp, 2008). Cet engouement pour la compétitivité des destinations touristiques va se traduire par de nombreuses recherches et travaux académique mais aussi par le développement de modèles opérationnels sur la question. 


\section{La compétitivité des destinations touristique dans la recherche académique:-}

Au niveau académique, notre examen de la littérature portant sur le sujet nous montre que de nombreux travaux de recherche ont porté sur la compétitivité touristique (Chon et al., 1991 ; Ritchie et Crouch, 1993; Hu et Ritchie, 1993; Gartner, 1993 ; De Keyser et Vanhove, 1994, Evans et Johnson, 1995 ; Dann, 1996 ; Pearce, 1997 ; Kozak et Rimmington, 1999 ; Crouch et Ritchie, 1999 ; Ritchie et Crouch 2000, Hassan, 2000 ; Mihalic, 2000 ; Thomas et Long, 2000 ; Gallarza et al., 2002 ; Dwyer et Kim, 2003; Kozak, 2002; Ritchie et Crouch 2003 ; Gonzalez et Falcon, 2003 ; Enright et Newton, 2004 ; Ruhanen, 2007 ; Cortes et al. 2007; Gomezel et Mihalic, 2008, Cracolici et Nijkamp, 2008.). Crouch l'une des références incontestée du domaine, a relevé dans sa revue de la littérature sur le sujet que l'analyse de la compétitivité des destinations touristiques ne peut se faire qu'en termes relatif et qu'elle peut viser l'étude d'un territoire particulier (par exemple Hong Kong pour Enright et Newton - 2004) ou l'analyse d'une des modalités de la compétitivité (la compétitivité prix pour Dwyer, Forsyth et Rao, 2002 ou le marketing de destination pour Buhalis, (2000) et Crouch, (2011).

Plusieurs constats peuvent être dégagés de cette littérature. Le premier est que ces travaux n'ont pas porté sur le développement d'un cadre théorique global définissant la compétitivité générale de la destination touristique mais ils se sont intéressés plus particulièrement à certaines destinations, à certaines formes de tourisme ou à certaines formes de tourisme dans certaines destinations. Le deuxième est la variation de l'échelle de destination retenue qui pouvait être des pays (ex : Omerzel Gomezel et Mihalic, 2008), des régions (ex : Cracolici et Nijkamp, 2008) ou des villes (ex : Clavers-Cortés et al., 2007). Le troisième est que certains travaux ont également eu des apports significatifs quant à l'opérationnalisation de la compétitivité touristique en proposant des méthodologies de mesure sophistiquées de cette compétitivité (ex : Enright et Newton, 2004). Le quatrième est que certains travaux de recherches ont porté sur des aspects particuliers de la compétitivité des destinations, y compris le positionnement des destinations (ex : Chacko 1998), les systèmes de gestion des destinations (ex: Baker, Hayzelden et Sussmann 1996), la commercialisation des destinations (ex : Buhalis 2000), la compétitivité des prix (ex :Dwyer, Forsyth et Rao 2000a, 2000b, 2000), la gestion de la qualité (ex : Go et Govers 2000), l'environnement (ex : Hassan 2000, Mihalic 2000), le tourisme de nature (ex :Huybers et Bennett 2003), la gestion stratégique (ex : Jamal et Getz 1996; Soteriou et Roberts 1998) et des voyages à forfait (ex :Taylor1995), l'image et l'attractivité des destinations au niveau touristique (ex : Dann, 1996; Chon et al., 1991 ; Hu et Ritchie, 1993 ; Gallarza et al., 2002 ; Gartner, 1993). Malgré ce foisonnement de travaux de recherche sur le concept de compétitivité des destinations touristiques peu d'auteurs ont tenté de lui donner une définition précise et les définitions qui existent ne montrent pas l'existence d'une définition standard, universelle et unanime. Ainsi, selon (Hassen, 2000), la compétitivité d'une destination touristique signifie «la capacité d'une destination à créer et intégrer des produits à valeur ajoutée en maintenant les ressources locales et préserver sa position sur le marché par rapport aux concurrents». Dans le même sens, Dwyer et al. (2011) retiennent que « la compétitivité d'une destination est liée à sa capacité à proposer des produits et services plus «performants » que ceux d'autres destinations pour satisfaire les besoins des visiteurs. ». De leur coté, Ritchie et Crouch (2000) la définissent comme «la capacité de la destination à créer de la valeur ajoutée et l'augmentation, par conséquent, du bien être national par la gestion des prestations et des processus en intégrant les relations entre eux dans un modèle économique et social». Adoptant une optique marketing D'Hauteserre (2000) parle de la capacité d'une destination de maintenir sa position sur le marché et de chercher l'amélioration au fils du temps afin de faire face à la concurrence. D'autres chercheurs comme Cracolici et Nijman (2008), Barney (1991); Grant, (2005) et Wernerfelt, 1984, sont allés s'attaquer à la question en se basant sur le mouvement des ressources et compétences pour expliquer et définir ce concept. Ces auteurs considèrent ainsi les destinations comme une combinaison de ressources physiques, naturelles, culturelles et humaines qui sont rares, inimitables et non-substituables et qui peuvent générer des compétences utiles pour créer et développer un avantage concurrentiel (Barney, 1991). Selon ces auteurs, la compétitivité d'une destination dépend de ses ressources tangibles et intangibles, de son management et de ses décisions.

De notre examen de la littérature académique, il parait bien évident que la compétitivité des destinations touristique est un sujet qui a retenu fortement l'attention des chercheurs mais il apparait également fort évident que les concepts de compétitivité en général et de compétitivité touristique en particulier, aisés à définir en apparence, posent d'importants problèmes de mesure (Cracolici et Nijkamp, 2008). Porter (1990) soulignait déjà l'ambigüité du concept de compétitivité. Du fait de l'ambiguïté et du caractère multiforme de ce concept, les auteurs ont ainsi utilisé des méthodologies diverses pour mesurer la compétitivité touristique. Kim (1998) notait déjà que les chercheurs, pour caractériser l'attractivité d'une destination, ont utilisé une grande variété de critères, même si certains d'entre eux étaient communs à la plupart des travaux. Dwyer et al. (2011) notent pour leur part qu'un problème majeur, caractérisant toute tentative d'établir des indices de compétitivité, est lié à l'intégration de critères 
«objectifs» de compétitivité (ex: évolutions des parts de marché, nombre d'emplois issus du tourisme...) et «subjectifs (ex : richesse de la culture, qualité du service, beauté des paysages...). Il n'existe pas ainsi actuellement de méthode unanimement reconnue permettant d'intégrer simultanément ces deux types de critères (Dwyer et Kim, 2003).

Les modèles de compétitivités touristiques:-

En réalité il n'existe pas à proprement dit de modèles de compétitivités qui ont été élaborés spécifiquement aux destinations touristiques mais certains chercheurs qui ont été aussi reliés par certains organismes internationaux voulant modéliser la compétitivité des destinations touristique, sont allés, sur la base des théories économiques de l'avantage comparatif et des travaux de Porter (1990) sur les avantages compétitifs, proposés des modèles génériques visant à cerner l'ensemble des particularités et des dimensions de la compétitivité des destinations touristiques. Nous nous contenterons dans ce point de présenter l'application du modèle de Porter et de présenter l'un des modèles les plus connu, le plus influent et dominant dans la littérature à savoir celui développé par Crouch et Richie en 1999.

\section{Le Diamant de M. Porter:-}

Le modèle mis au point par M. Porter (1990) dans son ouvrage la compétitivité des nations est celui qui va être plus tard appliqué au secteur du tourisme (THR - Barcelone). Porter affirme que le succès d'une entreprise ne dépend pas uniquement de sa stratégie ni de son positionnement, mais également de son intégration au sein de son environnement. Sa thèse centrale repose sur l'idée que les régions, les destinations, les « clusters » connaissent un certain succès au niveau d'activités ou de secteurs partiels, car leur environnement local est le plus dynamique et le plus stimulant et encourage les entreprises à préserver leur avantage. Il s'agit de la thèse centrale. Dans destinations touristiques il existe des groupes de sociétés directement ou indirectement liés au tourisme et qui concentrés sur une zone géographique spécifique et fonctionnent comme des clusters. La nature cosmopolite du secteur touristique, nécessitant différentes entreprises de taille et d'activités différentes mais liées par le même marché, stimule ce processus de regroupement. Dans son modèle Porter explique que la l'avantage concurrentiel d'une nation ou d'un nterritoire repose sur quatre facteurs interdépendants qui, ensembles, forment un « diamant » selon le terme utilisé par Porter pour faire référence à ces points déterminants. Pour Smeral (1996) un point de départ pour le développement de stratégies pour l'amélioration de la position concurrentielle d'une destination touristique est à chercher dans le système dynamique constitué par les facteurs déterminant développé par Porter. Ainsi par référence au modèle de porter nous pouvons bâtir les fondements de la compétitivité de la destination. Appliqué aux destinations touristiques le modèle se présentera comme suit : 
Fig. 1:- Points déterminants des avantages concurrentiels des destinations (Diamant de Porter).

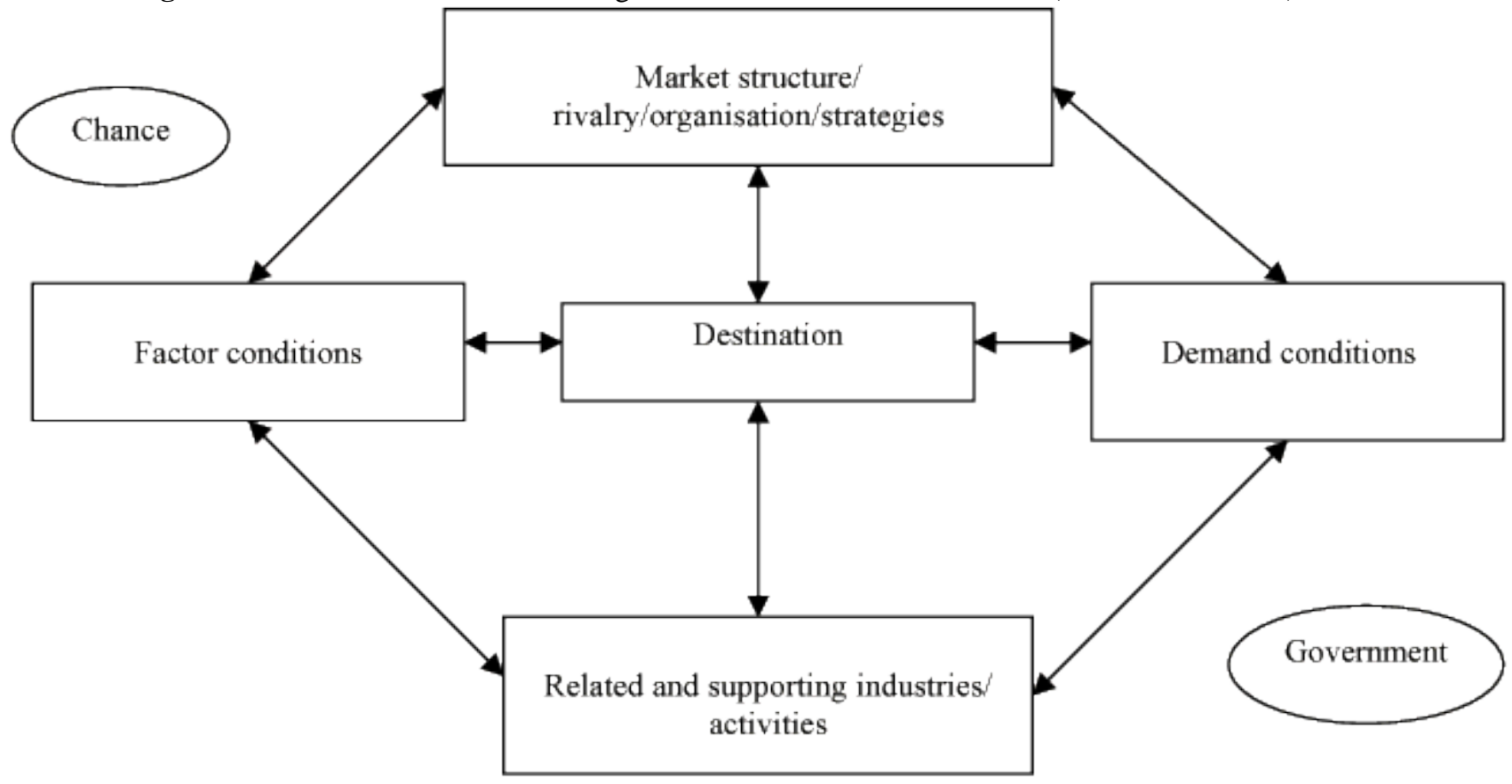

Ces points déterminants sont:-

1. Les conditions de facteurs. Renvoient à la détention de dotations factorielles naturelles, culturelles ou artificielles (attractions naturelles, ressources culturelles et historiques, infrastructures d'hébergement, de transport NTI,..., ressources humaines, prix des facteurs, efficacité de la production,...) fortes et nécessaires à la concurrence dans le secteur du tourisme et sans les quels il ne peut y avoir d'activité touristique.

2. Les conditions de la demande. Elément majeur et déterminant important de l'avantage concurrentiel les conditions de la demande renvoient pour d'une destination à plusieurs éléments dont principalement, la taille du marché local, la structure et la diversité du marché, la position sur les marchés à croissance rapide, le renforcement de la culture touristique des consommateurs et des sociétés d'accueil (Cooper et al., 2001), la protection du touriste-consommateur,...

3. Les secteurs d'activité associés et impliqués. La position concurrentielle d'une destination dépend et peut être renforcée également par la diversité et la qualité des fournisseurs installés dans le territoire (transporteurs, services connexes de haute qualité (système financier, de télécommunication, de santé), industrie agroalimentaire, installations sportives, de loisirs et culturelles, etc.

4. Structure du marché, rivalité, organisation et stratégie. Ces conditions sont très importantes et renvoient à la manière dont la destination et ses éléments sont organisés et gérés notamment la disponibilité d'un plan stratégique soutenu par toutes les parties impliquées, à la fois des secteurs public et privé, d'une stratégie marketing et promotionnelle claire et efficace, une image de marque forte, des mécanismes de coordination efficients, des organes de gestion bien structuré et gouvernés.

A ces quatre facteurs déterminants viennent s'ajouter deux autres facteurs non mois importants pour la compétitivité de la destination à savoir la chance et le gouvernement. Ces deux facteurs peuvent avoir une influence significative sur le degré de compétitivité de la destination et son succès notamment à travers d'une par les actions publiques qui peuvent être soit un facteur stimulant ou au contraire nuisant au développement du tourisme (ex : réglementation sur les hôtels, change, fiscalité, accueil et information des touristes, plan de circulation et d'aménagement, ...) et d'autre part, à travers la qualité de la gouvernance (implication ou non de toutes les parties prenantes).

A noter que le «diamant» se présente comme un système de renforcement mutuel dans le sens où il ya interdépendance entre les différents déterminants. Ainsi, par exemple, des conditions favorables de la demande n'engendreront un avantage concurrentiel que si l'état de la rivalité est suffisant pour que les entreprises (par ex., les hôtels) puissent leur répondre. De même, les avantages qui existent au niveau d'un point déterminant peuvent également créer ou améliorer les avantages d'un autre. 


\section{Le Modele De Crouch Et Richie:-}

Le modèle considéré le plus influent en matière de compétitivité touristique au regard des chercheurs et praticiens reste celui développé par Crouch et Ritchie en 1999. Ces deux auteurs, en se basant eux aussi sur le modèle du diamant de Porter mais en soulignant les limites des travaux de recherche précédents et des modèles généraux qui ont été élaborés sur la compétitivité des destinations qui selon eux malgré qu'ils ait produits une liste assez exhaustive des déterminants de la compétitivité des destinations ces déterminants n'ont pas tous une importance ou une influence égale pour cerner toute les dimensions concurrentielles de la destination. Ces auteurs estiment que le niveau de recherche sur la question de la compétitivité des destinations est assez maintenant développée pour pouvoir passer du simple listage des attributs et déterminants de cette compétitivité pour se recentrer sur l'étude de l'importance relative de chaque attribut et déterminant. Pour eux, l'impact d'un attribut de compétitivité sur la performance relative d'une destination est fonction à la fois de l'importance de l'attribut ainsi que du degré de variation des destinations sur l'attribut et bien qu'un attribut puisse être considéré comme important, il ne sera pas un déterminant de la compétitivité s'il ya peu de différence entre les destinations sur cet attribut. Sur fond de ces limites ils vont développer un modèle conceptuel de la compétitivité des destinations touristiques qui repose sur quatre composants majeurs : " les ressources et attractions centrales », «les facteurs et ressources supports », « le management de la destination » et les « déterminants». Les « ressources et attractions centrales » comprennent les éléments fondamentaux de l'attractivité d'une destination. Ce sont les raisons fondamentales qui expliquent pourquoi un visiteur choisit une destination plutôt qu'une autre. Les facteurs inclus dans cette composante sont la physiographie, la culture et l'histoire, les liens avec la destination, les activités, les événements et les infrastructures touristiques. La physiographie correspond au paysage et au climat, les liens avec la destination renvoient aux relations personnelles avec des résidents du pays émetteur et les infrastructures touristiques comprennent essentiellement le logement, les services de restauration, les moyens de transport et les attractions principales. L'image étant généralement considérée comme une composante importante de la compétitivité d'une destination, des attributs utilisés pour caractériser l'image comme par exemple le climat, les paysages ou l'hôtellerie, sont ainsi fréquemment utilisés pour mesurer la compétitivité touristique (Enright et Newton, 2004).

Les «facteurs et ressources supports» sont les facteurs susceptibles de soutenir le succès d'une destination au niveau touristique. Ils incluent notamment les infrastructures globales d'une destination, les établissements d'enseignement et les facteurs liés à l'accessibilité de la destination.

La troisième composante de la compétitivité touristique, à savoir le «management de la destination», se focalise sur les activités pouvant influencer les autres composantes, premièrement en améliorant l'attractivité des ressources et attractions centrales, deuxièmement en renforçant la qualité et l'efficience des facteurs supports et enfin en adaptant les contraintes imposées par la quatrième composante à savoir les «déterminants».

Alors que les aspects de cette composante les plus étudiés sont le marketing de la destination, des auteurs estiment qu'un spectre plus large d'activités liées au management devrait être pris en compte comme l'organisation et la maintenance des ressources et attractions clefs, ainsi que les services liés à ces ressources et attractions.

La quatrième composante est les « déterminants ». Elle inclut les facteurs qui peuvent modifier, éventuellement d'une manière négative, l'influence des trois autres composantes.

Ces facteurs sont par exemple la localisation, la structure des coûts et la sécurité. Ces facteurs ne sont pas contrôlés par les acteurs du tourisme mais peuvent jouer un rôle crucial dans la compétitivité d'une destination (Enright et Newton, 2004). Crouch et Ritchie (1999) prennent en compte également dans leur modèle de la compétitivité touristique le micro et le macro environnement de la destination qu'ils estiment être inter-reliés et affectant simultanément "le cour de la compétitivité" de la destination. Le microenvironnement renvoi aux aspects propres à la destination touristique et à ceux des acteurs principaux du tourisme dans la destination (T.O, Agences de voyages, les résidents, les touristes visiteurs, les employés des professions touristiques, les médias, les groupes de pression,...) pouvant faire l'objet de comparaison entre destinations concurrentes., les groupes d'action et de pression, les médias, les institutions financières et sociétés d'investissement, les administrations publiques, et l'environnement immédiat). Tandis que le macro-environnement concerne les éléments extérieurs à l'environnement immédiat de la destination mais pouvant exercer une certaine influence sur sa compétitivité. Il s'agit notamment, de l'attention portée par les autorités à l'environnement naturel et à sa protection, de la conjoncture économique internationale, des problèmes démographiques, de l'évolution technologique, etc. 
En définitive, nous pouvons retenir que le modèle de Crouch et Ritchie donne une indication claire sur les différents éléments sur lesquels repose la concurrence entre destinations touristiques et enseigne que la compétitivité et la supériorité d'une destination sur le marché touristique dépend de sa capacité à gérer et organiser ses ressources de manière rationnelle et efficace.

\section{Les Structures de Gestion des Destinations : Nature, Typoplogie et Fonctions:-}

Parallèlement au développement des travaux sur la compétitivité des destinations touristiques, la question des structures organisationnelles et de leur pertinence pour la compétitivité des destinations a été au centre de la recherche sur le tourisme. Ritchie et Crouch ayant discuté cette question en 1993 (Ritchie et Crouch, 1993), puis l'ayant reprise dans leur modèle en 2003, (Crouch et Richie, 2003) considèrent qu'à part dans le cas spécifiques des resorts, aucun prestataire et acteur du territoire de la destination ne peut prétendre répondre à lui tout seul à la demande des touristes et prendre lui-même en main leur expérience globale. Selon eux, comme système d'offre la destination, même s'elle ne constitue pas une entité commerciale légale, elle est composée d'un ensemble d'entreprises, d'organisations et de groupes qui œuvrent ensemble de manière semi-organisée, en partie coopérative, mais qui sont finalement motivés en grande partie par leur propre intérêt et doit être exploitée comme une entreprise (Ritchie et Crouch, 2003, p : 96). Dans cette perspective, les deux auteurs ont mis en lumière l'impact du management de la destination et le rôle de la Destination Management Organization (DMO) dans la compétitivité des destinations (Botti et Peypoch, 2011).

Après avoir jeté les éclairages nécessaires sur la problématique de la compétitivité des destinations touristiques et les défis qu'elle impose au management de la destination, nous chercherons dans cette section, toujours sur la base d'une recherche documentaire, comment la littérature aborde les structures de management des destinations et d'identifier le degré de correspondance et de concordance de ces structures et plus particulièrement de leur rôles et fonctions par rapport aux défis de la compétitivité soulevés et des exigences d'un management efficace des destinations touristiques.

Panorama des structures du management des destinations touristiques:-

Notre propos ici n'est pas de traiter des structures de gestion dans le cadre institutionnel distinguant les organes intervenants dans la gestion du tourisme aux échelles nationales, régionales et locale mais il s'agit de présenter et de discuter des structures de gestion des destinations touristiques telles qu'elles ont été définies et présentées dans le point précédent. L'examen de la littérature sur les structures de management des destinations touristiques nous montre qu'il existe cinq types de structures allant des structures basées sur le marché aux structures de type hiérarchique. Ces différents types de structures et leurs caractéristiques sont présentés dans le tableau suivant :

Tableau $\mathbf{n}^{\circ}$ 1:- Les structures idéales des destinations touristiques.

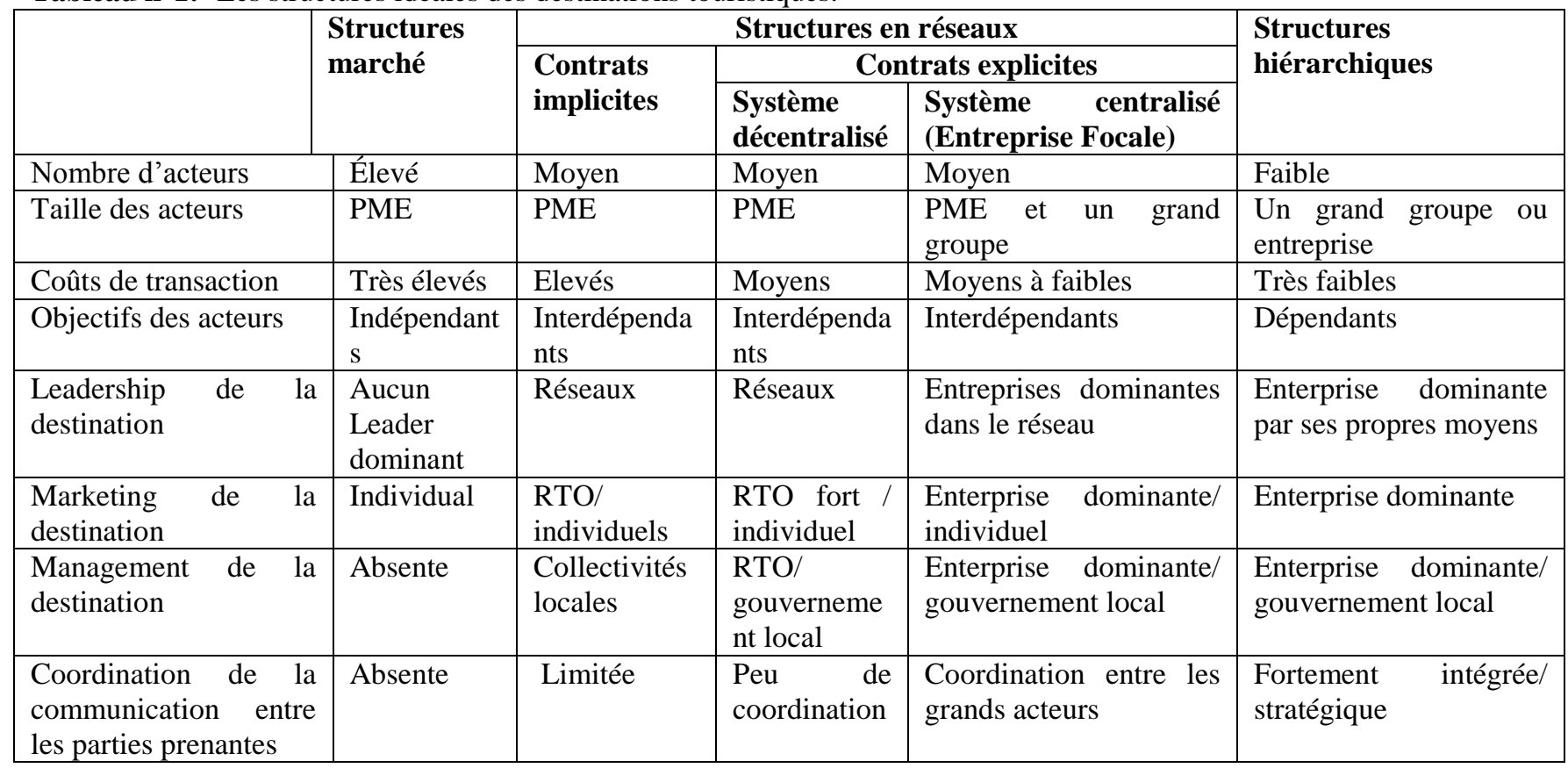


Source : Réalisé sur la base des travaux de Powel (1990) et de Bieger (2006)

Comme mentionné ce tableau distingue cinq types de structures de gestion des destinations allant de la structure du type marché, caractérisée par la présence d'un grand nombre d'entreprises pour l'essentiel de petites taille offrant individuellement leur services aux touristes, poursuivant leur propres objectifs, menant leur propres actions marketing et commerciales et se livrant à une concurrence intense sans aucune coordination à l'autre extrême de type corporate modèle dominant en Amérique du Nord et caractérisé par l'existence d'une société ou groupe privé («business corporation» selon Flagestad, 2001) dominant qui, en agissant au nom de la collectivité, cherche à atteindre des objectifs d'efficience et de profit à travers la réduction des coûts de transaction en internalisant tous les services nécessaires aux touristes et opère par intégration ou par contractualisation avec les autres sociétés de services qui sont généralement de petites tailles. Ce groupe détient le pouvoir politique de prise de décision en matière de gestion, de développement et de commercialisation de la destination et tous les autres acteurs et parties prenantes de la destination, y compris la sphère publique, dépendent de ce groupe qui opère à leurs égard par un marketing et une communication stratégiques et hautement intégrés. Entre ces deux types de structures extrêmes, il existe tout un continuum de configurations possibles épousant des formes de réseaux plus ou moins formalisé du point de vue opérationnel et de coordination. Dans ces formes en réseau la forme des contrats implicites caractérise les destinations dirigées par un grand nombre d'acteurs sous la coordination d'un organisme touristique régional (OTR). Dans ce type de structure, l'absence d'une réelle coordination formelle fait que les coûts de transaction restent assez élevés et le comportement coopératif des acteurs est dicté plus par la nécessaire interdépendance de leurs activités et la peur de sanctions en cas de concurrence déloyale et non par l'engagement volontaire dans une stratégie de coopération (Williams, Harkins et Latane, 1981). Egalement dans ce type de destination c'est en général l'autorité publique locale qui assure la fonction du management alors que le marketing et la commercialisation sont assurés par les entreprises et le cas échéant par l'organisation touristique régionale. Dans d'autres destinations les relations entre les différents acteurs peuvent être formalisées par une contractualisation explicite. Cette contractualisation favorise la coopération et la coordination réduisant les risques et les coûts de transaction et rendant la gestion et la commercialisation de la destination plus efficace (Granovetter, 1973). Ce type de structure admet deux principales variantes. Soit un système de nature décentralisé dans le quel les entreprises, dont la grande majorité sont des PME, se constituent en réseau qui prend en charge la gestion et la promotion de la destination en s'aidant ou non par organisme touristique régional. Soit un système de nature centralisé avec la présence d'une entreprise dominante qui peut mobiliser assez de ressources et de pouvoir et qui joue le rôle de leadership et fixe les stratégies de développement et de promotion de la destination qui sont suivies par les autres acteurs. Dans ce type de structure étant donné que cet acteur stratégique ne peut à lui tout seul assurer tous les services et toute l'expérience aux touristes et visiteurs il reste dépendant en partie des autres prestataires avec lesquels il doit collaborer et coordonner les actions de communication et de marketing.

\section{Fonctions principales de gestion des destinations:-}

En retenant uniquement les travaux de recherche et les études qui ont porté sur le management des destinations, les principales fonctions de ce management répertoriées selon l'ordre de leur nombre et fréquence de citation par les différents auteurs sont reprises dans le tableau suivant. 
Tableau $\mathbf{n}^{\circ}$ 2:- Fonctions du management de la destination.

\begin{tabular}{|c|c|}
\hline Fonctions & Auteurs \\
\hline $\begin{array}{l}\text { Marketing de la destination, branding et } \\
\text { positionnement }\end{array}$ & $\begin{array}{l}\text { Aberg (2014), Anderson (2000), Crouche et Richie (1999), Harril } \\
\text { (2005), Jamieson (2006), Lasser et Beritelli (2013), Longjit et Pearce } \\
\text { (2013), Morrisson (2013), Pearce et Schänzel (2013), Pearce } \\
\text { (2014b), Pechlaner, herntrei et Kofink (2009) et OMT (2007) }\end{array}$ \\
\hline $\begin{array}{l}\text { Planification, suivi et évaluation des } \\
\text { destinations }\end{array}$ & $\begin{array}{l}\text { Aberg (2014), Anderson (2000), Crouche et Richie (1999), Dweyr et } \\
\text { Kim (2003), Fuchs et Weiermair (2004), Jamieson (2006), Lasser et } \\
\text { Beritelli (2013), Morrisson (2013), Pearce (2014b), Ryglová (2008) } \\
\text { et OMT (2007). }\end{array}$ \\
\hline Développement des produits/services & $\begin{array}{l}\text { ARTN (n.d), Ivanis (2011), Jamieson (2006), Lasser et Beritelli } \\
\text { (2013), Morrison (2013), Pearce (2014b), Pechlaner et al (2009), } \\
\text { Risteski, Kocevski et Arnaudove (2012) et OMT (2007). }\end{array}$ \\
\hline $\begin{array}{l}\text { Recherche, gestion de l'information et } \\
\text { renforcement des connaissances }\end{array}$ & $\begin{array}{l}\text { Anderson (2000), ATRN (n.d.), Crouch et Richie (1999), Harrill } \\
\text { (2005), Morrison (2013), Pavlovitch (2003), Pearce (2014b) et OMT } \\
\text { (2007). }\end{array}$ \\
\hline $\begin{array}{l}\text { Gestion des ressources, gestion de } \\
\text { l'environnement }\end{array}$ & $\begin{array}{l}\text { Crouch et Richie (1999), Dwyer et Kim (2003), Fuchs et Weiermai } \\
\text { (2004), Longjit et Pearce (2013), Pavlovitch (2003), Risteski et al. } \\
\text { (2012) et Ryglová (2008). }\end{array}$ \\
\hline $\begin{array}{l}\text { Gestion des visiteurs, gestion de } \\
\text { l'expérience des visiteurs, gestion des } \\
\text { risques d'aventure, gestion de la sécurité }\end{array}$ & $\begin{array}{l}\text { Anderson (2000), Crouch et Richie (1999), Longjit et Pearce (2013), } \\
\text { Pavlovitch (2003), Pearce et Schänzel (2013) et Risteski et al. (2012) }\end{array}$ \\
\hline Construction de relations & $\begin{array}{l}\text { Crouch et Richie (1999), Dwyer et Kim (2003), Jamieson (2006), } \\
\text { Morrison (2013) et OMT (2007). }\end{array}$ \\
\hline $\begin{array}{l}\text { Développement des ressources humaines, } \\
\text { formation }\end{array}$ & $\begin{array}{l}\text { Dwyer et Kim (2003), Longjit et Pearce (2013), Pearce (2014b) et } \\
\text { OMT (2007). }\end{array}$ \\
\hline $\begin{array}{l}\text { Responsabilité organisationnelle, leadership } \\
\text { et partenariat }\end{array}$ & $\begin{array}{l}\text { Anderson (2000), Crouch et Richie (1999), , Jamieson (2006), } \\
\text { Risteski et al. (2012) }\end{array}$ \\
\hline Lobbying & Lasser et Beritelli (2013) \\
\hline Coordination du service & Lasser et Beritelli (2013), \\
\hline Fourniture et diffusion d'Informations & Pearce et Schänzel (2013) \\
\hline $\begin{array}{l}\text { Régulation et canalisation de la pression } \\
\text { touristique }\end{array}$ & Laws (1995) \\
\hline Gestion du cycle de vie & Sainaghi (2006) \\
\hline $\begin{array}{l}\text { Gestion de problèmes particuliers (par } \\
\text { exemple, capacité de charge) }\end{array}$ & Sainaghi (2006) \\
\hline Soutien aux entreprises & OMT (2007) \\
\hline
\end{tabular}

Source: Elaboré sur la base des travaux de Douglas G. Pearce (2015)

De la lecture de ce tableau nous pouvons relever en premier que le management des destinations touristiques, compte tenu de la complexité et de la multi-dimensionnalité de celle-ci exige un grand nombre de taches et de fonctions portant sur plusieurs aspects. Ces fonctions, telles qu'elles ressortes de ces travaux et études, vont du marketing et de la commercialisation, à la planification de développement de la destination et de la formulation des stratégies, à la gestion des ressources et de l'environnement, au développement de l'offre et des produits et activités, la recherche et l'innovation, le suivi et l'évaluation et d'autres éléments spécifiques, la gestion de l'information et des connaissances, la gestion des touristes et visiteurs mais aussi à la gestion et de la collaboration et de la coordination entre les différents acteurs et les différentes parties prenantes agissant dans le territoire de la destination. Certaines autres dimensions aussi importantes dans la gestion des destinations ont été peu citées par les auteurs, tel est le cas du lobbying, l'information des touristes et visiteurs, la gestion du cycle de vie de la destination, la gestion de la capacité de charge, la régulation et la canalisation de la pression touristique et le soutien aux entreprises des différentes branches du secteur touristiques.

L'autre constat fondamental à relever de cette lecture de la littérature sur la question est que malgré l'existence d'un certain accord entre les chercheurs sur nombre de fonctions du management de la destination il reste qu'aucun de 
ces travaux et de ces recherches n'a discuté ni la question de la pertinence, de la viabilité et de l'efficacité de telle ou telle de ces fonctions, ni celles qui sont effectivement réalisées dans les destinations.

\section{Les fonctions de l'organisme de gestion de destination (OGD ou DMO):-}

Plusieurs autres chercheurs se sont concentrés non pas uniquement sur les fonctions du management de la destination mais sont allés se poser des interrogations sur celles qui devraient être assurées par les organes ou structures de gestion de la destination, appelées généralement Organisme de Gestion des destinations (OGD) ou par son équivalent anglais Destination Management Organisation (DMO). Recherchant à identifier les rôles, formes et fonctions de ces structures, les auteurs se sont heurtés en premier lieu à la problématique de cette appellation de DMO. En effet, dans sa conception anglo-saxonne le ' $M$ ' dans le terme DMO renvoi au marketing plutôt qu'au management. Alors que dans son acception la plus courante, notamment celle de l'OMT, le terme DMO est désigné comme Organisme de Gestion des Destinations. D'autres auteurs sont mêmes allés utiliser les deux acceptations pour parler d'organisme de gestion et de marketing des destinations. Il est à noter qu'au fond cette querelle entre auteurs ne porte pas sur le coté sémantique du terme mais plutôt sur l'intérêt de préciser exactement ce que reflète l'appellation en termes de fonctions et d'activités de base assurées par cette organisation dans le cadre d'une destination. Comme pour le cas des fonctions du management des destinations nous présenterons les fonctions de l'OGD de la destination en fonction de l'ordre et fréquence de citation par les différents auteurs. Les résultats de notre lecture de la littérature sur cette question sont repris dans le tableau suivant.

Tableau n ${ }^{\circ}$ 3:- Les fonctions de l'OGD à l'aune de la literature.

\begin{tabular}{|c|c|}
\hline Fonctions & Auteurs \\
\hline $\begin{array}{l}\text { Marketing de la destination, Branding et } \\
\text { positionnement }\end{array}$ & $\begin{array}{l}\text { Baggio (2008), Bieger et al (2009), Bornhorst, Ritchie et } \\
\text { Sheehan, Bramwell et Rawding (1994), Crouch et Richie (1999), } \\
\text { Elbe et al (2009), Gretzel et al (2006), Heath et Wall (1999), } \\
\text { Osmankovic et al (2010), Pechlaner et al. (2012), Presenza et al. } \\
\text { (2005), Prideaux et Cooper (2002), ROS Développement et } \\
\text { planification (2008), Sheeban, Richie et Hudon (2007) et Socher } \\
(2000))\end{array}$ \\
\hline $\begin{array}{l}\text { Renforcement des relations / coordination / } \\
\text { facilitation }\end{array}$ & $\begin{array}{l}\text { Bornhorst et al. (2010), Crouch et Richie (1999), Elbe et al. } \\
\text { (2009), Heath et Wall (1992), Presenza et al. (2005), Prideaux et } \\
\text { Cooper (2002), Sheehan et al (2007) et OMT (2007) }\end{array}$ \\
\hline $\begin{array}{l}\text { Développement des produits/ développement } \\
\text { des activités }\end{array}$ & $\begin{array}{l}\text { Baggio (2008), Bieger et al. (2009), Bornhorst et al. (2010), } \\
\text { Osmankovic et al (2010), Pechlaner et al. (2012), Socher (2000) } \\
\text { et OMT (2007) }\end{array}$ \\
\hline $\begin{array}{l}\text { Planification, formulation de la stratégie, suivi } \\
\text { et évaluation }\end{array}$ & $\begin{array}{l}\text { Baggio (2008), Bornhorst et al. (2010) , Heath et Wall (1992), } \\
\text { Pechlaner et al. (2012), Jenkins et al (2011) et OMT (2007). }\end{array}$ \\
\hline $\begin{array}{l}\text { Management des ressources, management de } \\
\text { l'environnement }\end{array}$ & $\begin{array}{l}\text { Bornhorst et al. (2010), Presenza et al. (2005) et ROS } \\
\text { Développement et planification (2008) }\end{array}$ \\
\hline $\begin{array}{l}\text { Développement des ressources humaines, } \\
\text { Formation }\end{array}$ & $\begin{array}{l}\text { Presenza et al. (2005), ROS Développement et planification } \\
(2008) \text { et OMT (2007). }\end{array}$ \\
\hline Management de la destination & $\begin{array}{l}\text { Bornhorst et al. (2010), Crouch et Richie (1999) et Gretzel et al } \\
(2006) \text {. }\end{array}$ \\
\hline Assurance qualité & $\begin{array}{l}\text { Osmankovic et al (2010) et ROS Développement et planification } \\
(2008) \text {. }\end{array}$ \\
\hline Information et réservations & $\begin{array}{l}\text { Bieger et al (2009), ROS Développement et planification (2008) } \\
\text { et OMT (2007). }\end{array}$ \\
\hline $\begin{array}{l}\text { R\&D, Système d'information, management et } \\
\text { développement des connaissances }\end{array}$ & Osmankovic et al (2010) et Presenza et al. (2005) \\
\hline $\begin{array}{l}\text { Gestion des visiteurs, gestion de l'expérience } \\
\text { clients }\end{array}$ & Bornhorst et al. (2010) et Presenza et al. (2005) \\
\hline Fourniture de services, coordination & $\begin{array}{l}\text { Bornhorst et al. (2010) et ROS Développement et planification } \\
(2008) \text {; }\end{array}$ \\
\hline Soutien aux entreprises & $\begin{array}{l}\begin{array}{l}\text { Prideaux et Cooper (2002) et } \\
\text { planification (2008) }\end{array} \\
\end{array}$ \\
\hline Prise de décisions ou exécution & Baggio (2008) et Bornhorst et al. (2010) \\
\hline
\end{tabular}




\begin{tabular}{|l|l|}
\hline Management des crises & Presenza et al. (2005) \\
\hline Aide à l'accès au financement & Presenza et al. (2005) \\
\hline Améliorer le bien-être des résident & Bornhost et al. (2010) \\
\hline Animation & ROS Développement et planification (2008) \\
\hline
\end{tabular}

Source: Elaboré sur la base des travaux de Douglas G. Pearce (2015)

Nous pouvons à la lumière de ce tableau constater que la fonction marketing de la destination se distingue comme le propre du travail et de la mission d'un OGD, suivie de l'établissement des relations et de la coordination entre acteurs et par la suite de l'activité développement des produits et de l'offre. Les autres taches relatives à la planification, la stratégie de développement de la destination, la gestion des ressources, le soutien aux entreprises et l'aide à l'accès au financement,..., ne sont que peut traitées par les différents auteurs quand il s'agit du rôle et des fonctions de l'OGD. Cela nous amène à formuler la conclusion que, malgré qu'elle est citée par quelques auteurs, la fonction du management de la destination apparait comme une fonction distincte ne relevant pas des attributions d'un OGD.

Un autre constat à relever de ces études est que, comme pour le cas du management des destinations, hormis quelques tentatives, les auteurs sont restés muets sur les fonctions effectives exécutées par les OGD et se sont plus intéressés à ce qu'ils devraient ou pourraient faire. Les quelques études empiriques menées en Amérique du Nord et en Europe (Presenza, Sheehan et Ritchie,2005 ; Getz et al., 1998 et Gretzel et al., 2006, ROS Development and Planning, 2008) ont toutes montré que l'amalgame entre la fonction du marketing de la destination et l'intégration de la gestion de la destination par un OGD ont abouti à l'échec jusqu'au point où Pike et Page (2014, p. 205) sont allés affirmer que: «L'utilisation de l'organisation de gestion des destinations comme un descripteur générique est inutile dans l'ajout de clarté et de but à la discussion du rôle des OGD, car il confond le besoin perçu de gestion avec la fonction largement de commercialisation qu'ils entreprennent effectivement... un DMO est un organisme responsable de la commercialisation d'une destination touristique identifiable avec une frontière géographique explicite». Cette affirmation nous montre une fois encore que la fonction de gestion de la destination et à distinguer de celle du marketing de la destination et donc elle ne fait pas partie de la mission et des attributions de l'OGD. Cette conclusion contredit aussi la conception de l'OMT qui dans l'une de ses publications a défini l'OGD comme "une entité de premier plan qui coordonne la gestion de tous les éléments intégrant une destination (lieux d'intérêt, équipements, facilités d'accès, moyens de transport, hébergements, etc.) et des activités telles que le développement de produits, leur marketing et l'établissement de leurs prix. L'OGD fait le lien entre les diverses autorités, les parties prenantes et les groupes d'intérêt. Il facilite également les partenariats et les synergies dans le cadre d'une stratégie cohérente visant à améliorer le développement, la gestion et la promotion de la destination. Il existe de nombreux types d'OGD en fonction des besoins et des spécificités de chaque destination à l'échelon national, régional et/ou local." (OMT, 2007)

A ce stade est sur la base des conclusions de notre lecture de la littérature existante sur la question, il nous semble pertinent de se poser la question sur qui devrait assurer et exercer les fonctions de gestion de la destination?

Certains éléments de réponse à cette interrogation sont à chercher dans les travaux et études d'un certains nombre d'autres chercheurs qui ont essayé d'étudier la question en interpellant le rôle joué par la sphère publique dans le développement et la gestion des destinations. En effet, ces auteurs mettant l'accent sur la prise de conscience, par les acteurs publics territoriaux, de l'importance du tourisme pour le développement et la promotion des territoires et régions et leur implication de différentes manières dans le secteur (Aménagement, réalisation d'infrastructures, élaboration de stratégies, gestion de biens collectifs, incitations et soutien aux investisseurs et aux entreprises,...) ont commencé à s'intéresser aux fondements, formes et modalités de cette implication et aux rôles et fonctions que devrait assurer cette sphère publique dans le management de la destination et le degré de leur efficacité. L'intérêt porté à cette nouvelle variable va élargir le champ des investigations sur la gestion des destinations touristiques du simple contexte sectoriel et professionnel vers un contexte plus large intégrant tous les acteurs et toutes les parties prenantes présents sur le territoire de la destination. Ainsi, une importante littérature va se développer sur les modalités juridiques de cette gestion (Dredge et al., 2011 et Jenkins et al., 2011), les fonctions à assurer par la sphère publique (Bramwell et Rawding, 1994), le partage des responsabilités (Longjit et Pearce, 2013) et la problématique de la coordination (Palmer,1996) et de la gouvernance (Barrado Timón, 2004, Gerbaux et Marcelpoil, 2006 et Marsat et al. , 2010), de la durabilté (Dredge et al. 2011). Toutefois, la littérature note que, mise à part l'étude de Marsat et al. (2010), qui ont pu développer une approche globale et multifonctionnelle de la gestion de la destination et ont examine les liens entre les fonctions de la sphère publique locale et le management territorial, le produit de ce 
courant de pensée est resté très diffus et cloisonné sur les fonctions spécifiques (marketing et commercialisation, aménagement, développement durable,...) et n'a pu se soulever au stade d'une véritable théorie de gestion des destinations.

\section{Conclusions et implications theoriques:-}

Notre revue de la littérature nous a montré que la question de la compétitivité des destinations touristiques est une question centrale dans les travaux de recherche en tourisme. Toutefois, les concepts de destination, de compétitivité que de compétitivité de destination, malgré la foisonnante littérature de recherche qui leur a été consacrée restent encore entachés d'un certain flou et ambigüité. La compétitivité de la destination apparait ainsi comme la finalité recherchée mais qui partage encore les chercheurs et les organismes intéressés par le tourisme sur les dispositifs et les stratégies à mettre en œuvre ainsi que les formes et les styles de gestion à adopter pour la booster. Une première réalité qui émerge clairement de cette littérature est que face aux défis imposés par la compétitivité le management de la destination touristique n'est pas une tache aisée et nécessite une multitude de fonctions et fait intervenir un grand nombre d'acteurs. Vu comme système, la destination est composé d'un complexe d'acteurs indépendants mais en même temps interdépendantes dans leurs activités et participant tous à la délivrance de l'expérience aux touristes et aux visiteurs et générant de la valeur pour le territoire et ses différentes parties prenantes. L'autre conclusion majeure qui se dégage de notre examen de la littérature est l'absence d'un modèle unique et unanime relatif aux fonctions à assurer et les structures qui devraient les assurer. En effet, nous avons ainsi pu relever que si les chercheurs identifient une palette très large de fonctions, taches et activités que nécessite le management de la destination, ils ne reconnaissent qu'un nombre limité de ces fonctions, taches et activités lorsqu'ils aborde les organismes de gestion des destination ou la sphère publique. S’il est reconnu que les défis imposés par la compétitivité exigent pour une destination touristique la nécessité de coordonner et d'intégrer de multiples fonctions et d'acteurs aux intérêts fortement divergents, il est aussi de mise de relever la grande variété des situations et des types de destinations touristiques et des contexte de leur émergence et développement et la nature et le nombre des acteurs impliqués dans leur gestion et promotion qui sont autant de contraintes pour les chercheurs les poussant à adopter des postures et des approches différentes ou de se concentrer sur certains aspects plutôt que d'autres.

La troisième conclusion importante à tirer de cette synthèse de la littérature et qui est directement liée à notre problématique principale de cette recherche est que les principaux défis imposés par la compétitivité même s'ils ne semblent pas être des préoccupations primordiales parmi les fonctions des structures de management des destinations touristiques comme c'est le cas par exemple des fonctions marketing, sont plus cités comme des fonctions de management de la destination et dans une moindre mesure parmi celles de l'OGD. Nous pensons que les considérations relatives à l'expérience client, à l'image de marque, au numérique, à la gestion des connaissances et au développement durable, malgré qu'elles soient au centre des préoccupations de nombreux chercheurs et qui les considèrent comme des déterminants de l'attractivité et de la compétitivité des destinations touristiques, elles sont encore insuffisamment modélisées pour le cas de ces dernières et qu'il s'agit de préoccupations incombant à toutes les parties prenantes de la destination prise comme système ou comme une entreprise virtuelle au sens de Botti (Botti, 2009).

En définitive, nous pensons que l'absence d'un corpus théorique complet et bien établi, aussi bien sur la gestion et la compétitivité des destinations touristiques que plus particulièrement sur les structures de gestion et leurs fonctions, traduit le caractère récent de l'intérêt pour ces domaines de la part de la science du management et dénote de leu caractère complexe et multidimensionnel toutefois, ils laissent aussi la porte grandement ouverte pour les chercheurs pour développer des recherches et études plus poussées sur les différentes dimensions du management stratégique et opérationnel des destinations touristiques. 


\section{References:-}

1. Anderson, D. (2000). Destination management. In: J. Jafari (Ed.), Encyclopedia of Tourism (p. 146) New York: Routledge.

2. Beritelli, P., Bieger, T. et Laesser, C., (2007), «Destination governance: Using corporate governance theories as a foundation for effective destination management», Journal of Travel Research, vol. 46, no. 1, pp. 96-107, 2007.

3. Beritelli, P., Bieger, T., et Laesser (2014). The new frontiers of destination management: applying variable geometry as a function-based approach. Journal of Travel Research, 53(4), 403-417.

4. Bieger, T., Beritelli, P. et Laesser, C. (2009). Size matters! Increasing DMO effectiveness and extending tourism destination boundaries. Tourism, 57(3), 309-327.

5. Bodega, D., Cioccarelli, G., et al. (2004). New inter-organizational forms: evolution of relationship structures in mountain tourism. Tourism Review, 59(3), 13-19.

6. Botti, L., Peypoch, N. (2012) . De la touristicité des territoires. Tourism \& Territories, 68-100.

7. Botti, L., Peypoch, N. et Solonandrasana, B. (2009). Tourism destination competitiveness: the French regions case. European Journal of Tourism Research, 2(1), 5-24.

8. Bornhorst, T., Brent Ritchie, J. R., et Sheehan, L. (2010). Determinants of tourism success for DMOs \& destinations: an empirical examination of stakeholders' perspectives. Tourism Management, 31(5), 572-589.

9. Bruno, I. (2009). Marketing expérientiel: Comment concevoir et stimuler l'expérience client. Dunod.

10. Buhalis, D. (2000). Marketing the competitive destination of the future. Tourism Management, 21(1), 97-116.

11. Boualemn, K. et al., (2011). Le concept de destination Diversité sémantique et réalité organisationnelle. TÉOROS, vol. 30, no 1, p. 12-24

12. Boston Consulting group, 1988. Les mécanismes fondamentaux de la compétitivité. In La logistique d'entreprise, vers un management plus compétitif, Dunod, Paris.

13. Buhalis, D. (2000), «Marketing the competitive destination of the future », Tourism Management, 2 , pp. 97116.

14. BUTLER, Richard (1980) "The concept of a tourist area cycle of evolution : implications for management of resources", The Canadian Geographer, no 24, vol 1, p. 5-12.

15. Chon (1990) "The rôle of destination image in tourism : A review and discussion", Revue du tourisme, no 2, p. 2-9.

16. Cracolici M. F., et Nijkamp P. (2009). The attractiveness and competitiveness of tourist destinations: a study of southern Italian regions. Tourism Management, 30(3), 336- 344.

17. Cooper, C., Scott, N. and Baggio, R. (2009). Network position and perceptions of destination stakeholder importance', Anatolia, Vol. 20 No. 1, pp. 33-45.

18. Crouch G. I., et Ritchie J. R. (1999). Tourism competitiveness and societal prosperity. Journal of Business Research, 44(3), 137-152.

19. Davidson, R. et Maitland, R. (2002). Tourism destinations, the dynamics and evolution of tourism destination. London: Hodder and Stoughton. $281 \mathrm{p}$.

20. De Keyser R. et Vanhove N. (1994). The competitive situation of tourism in the Caribbean areaMethodological approach, Revue de Tourisme, 3, pp. 19-22.

21. Demen-Mayer, C. (2005). Le tourisme : essai de définition. Management \& Avenir, 1(3), 7-25.

22. Dwyer, L., et Kim, C. (2003). Destination competitiveness: determinants and indicators. Current Issues in Tourism, 6(5), 369-414.

23. D'Angella, F., De Carlo, M. et Sainaghi, R. (2010). Archetypes of destination governance: a comparison of international destinations. Tourism Review, 65 (4), 61-73.

24. Evans M. R. \& Johnson R.B.(1995).Identifying competitive strategies for successful tourism destination development, Journal of Hospitality and Leisure Marketing, 31 : 1, pp. 37-45.

25. Gerbaux, F., et Marcelpoil, E. (2006). Gouvernance des stations de montagne en France: les spécificités du partenariat public-privé. Revue de Géographie Alpine, 94(1), 9-19.

26. Hassan, S.S. 2000 Les déterminants de la compétitivité du marché dans une industrie du tourisme écologiquement durable. Journal of Research Voyage, 38 (3): 239-245 Hazbroucq, 2007

27. Hamel G. et Prahalad C.K (1994). Competing for the future, Harvard Business Review, juillet-août

28. Lachaal L. (2001). La competitivité : Concepts, définitions et applications. CIHEAM, 2001. p. 29-36 (Cahiers Options Méditerranéennes; n. 57)

29. Laesser, C. et Beritelli, P. (2013). St Gallen consensus on destination management. Journal of Destination Marketing \& Management, 2, 46-49. 
30. Langlois, M., (2004). «Le Management Expérientiel Médiatique (MEM) des organisation de services». Glec.

31. Laurent Botti, (2010). Pour une gestion de la touristicité des territoires : stratégie collective et management de l'attractivité », Mondes du Tourisme. Mis en ligne le 30 septembre 2015, consulté le 02 mars 2017. URL : http://tourisme.revues.org/325.

32. Lawrence, P.R. et Lorsh, J.W. (1986). Organization and environment: managing differentiation and integration. Harvard Business School Clasics.

33. Laws, E. (1995). Tourist destination management: issues, analysis and policies. London: Routledge. LozAto-Giotart et BALFET,M. (2004). Management du tourisme. Pearson Education.

34. Marniesse S. et Filipiak E. (2003). Compétitivité et mise à niveau des entreprises : Approches théoriques et déclinaisons opérationnelles. Agence Française de Développement, Paris.

35. Marsat et al. (2010). Vers une analyse multi-acteurs, multi-fonctions, du management stratégique de destination touristique - deux études de cas. 3ème Journée de Recherche et d'Echanges en Management du Tourisme,

36. Marsat, J.-B., Guerra, F. et Lepinay, T. (2010). Management stratégique de destination touristique et management territorial: le cas du Massif du Sancy. Colloque Management environnemental d'une destination touristique - Un facteur de compétitivité du tourisme. Gestion du tourisme 21 (1): 65-78

37. MIT (2000). La mise en tourisme des lieux : un outil de diagnostic. Mappemonde, 57(1), 2-6.

38. OCDE (2013), «Indicateurs de la compétitivité du tourisme : Document d'orientation », Éditions OCDE. http://dx.doi.org/10.1787/5k43jc9mnrxw-fr

39. Pearce, D. G. (2014). Towards an integrative conceptual framework of destinations. Journal of Travel Research, 53(2), 141-153

40. Pike, S. (2004). Destination marketing organizations. Oxford, UK: Elsevier.

41. Porter, M. (1990). The competitive advantage of nations. Harvard Business Review, 68(2), 73-93

42. .Ritchie, J. R. B. Crouch, G. 2000 La destination compétitive: une perspective de développement durable. Dans: Tourism Management, vol. 21 (1): 1-7

43. Ritchie J.R.B. et Crouch G. I., (2003). The competitive destination: A sustainable tourism perspective. Cambridge », Cabi Publishing.

44. Ruhanen, L. (2013). Local government: facilitator or inhibitor of sustainable tourism development. Journal of Sustainable Tourism, 21(1), 80-98.

45. Ruhanen L., (2007) .Destination competitiveness. In A. Matias, P. Nijkamp et P. Neto (Eds.), Advances in modern tourism research, Heidelberg: Physika-Verlag, pp. 133-152.

46. Sainaghi, R. (2006). From contents to processes: versus a dynamic destination management model (DDMM). Tourism Management, 27(5), 1053-1063.

47. Tixier D. et Mathe H. (1996). La logistique d'entreprise, vers un management plus compétitif, Dunod, Paris.

48. Tocquer, G. et ZINS, M. (1999). Marketing du tourisme, deuxième édition, Paris : Gaëtan Morin.

49. Van Der Yeught, C., «Favoriser l'émergence d'un acteur stratégique dans les destinations touristiques pour répondre aux défis du développement durable », Management \& Avenir, 2009/6 (n²6) Pages 300 - 317).

50. VIOLIER, Philippe (2009). Proposition pour un modèle d'analyse et de management des lieux touristiques. Éditions Téoros, Presses de l'Université du Québec.

51. Zhang at al. (2011). The evaluation of tourism destination competitiveness by TOPSIS \& information entropy A case in the Yangtze River Delta of China. Tourism Management, 32, 443-451. 